\title{
“Referred Visual Sensations": Rapid Perceptual Elongation after Visual Cortical Deprivation
}

\author{
Daniel D. Dilks, ${ }^{1}$ Chris I. Baker, ${ }^{2}$ Yicong Liu, ${ }^{1}$ and Nancy Kanwisher ${ }^{1}$ \\ ${ }^{1} \mathrm{McG}$ overn Institute for Brain Research, Massachusetts Institute of Technology, Cambridge, Massachusetts 02139, \\ and ${ }^{2}$ Laboratory of Brain and Cognition, National Institute of Mental Health, National Institutes of Health, Bethesda, Maryland 20892
}

Visual perceptual distortion (i.e., elongation) has been demonstrated in a single case study after several months of cortical deprivation after a stroke. Here we asked whether similar perceptual elongation can be observed in healthy participants after deprivation and, crucially, how soon after deprivation this elongation occurs. To answer this question, we patched one eye, thus noninvasively and reversibly depriving bottom-up input to the region of primary visual cortex (V1) corresponding to the blind spot (BS) in the unpatched eye, and tested whether and how quickly elongation occurs after the onset of deprivation. Within seconds of eye patching, participants perceived rectangles adjacent to the BS to be elongated toward the BS. We attribute this perceptual elongation to rapid receptive field expansion within the deprived V1 as reported in electrophysiological studies after retinal lesions and refer to it as "referred visual sensations" (RVS). This RVS is too fast to be the result of structural changes in the cortex (e.g., the growth of new connections), instead implicating unmasking of preexisting connections as the underlying neural mechanism. These findings may shed light on other reported perceptual distortions, as well as the phenomena of "filling-in."

\section{Introduction}

Perceptual distortion after deprivation has been well documented in the human adult somatosensory system. For example, stimulation of the face in arm amputees produces sensation not only on the face but also on the missing arm (Ramachandran et al., 1992). Researchers have attributed these "referred sensations" to receptive field expansion of deprived somatosensory neurons (for review, see Calford, 2002), arguing that, when the arm region of somatosensory cortex is deprived, the deprived "arm" neurons begin responding to stimuli that normally activate the adjacent "face" neurons. These referred sensations have been found in $<24 \mathrm{~h}$ after amputation (Borsook et al., 1998) and less than $1 \mathrm{~h}$ after pharmacological nerve block (Weiss et al., 2004).

In contrast to the somatosensory system, there has been little work on the perceptual consequences of deprivation in the visual system and no work investigating the time course of any such consequences. However, one study reported visual distortion after deprivation as a result of stroke (Dilks et al., 2007). The patient's stroke spared primary visual cortex (V1) but destroyed the optic radiation fibers that normally provide input to $\mathrm{V} 1$ from the upper left visual field (LVF). As a consequence, the stroke patient was blind in the upper LVF, and stimuli presented in the lower LVF were vertically elongated (e.g., a square pre-

Received April 1, 2009; revised May 19, 2009; accepted June 10, 2009.

This work was supported by National Institutes of Health Grant EY016559 (N.K.), Kirschstein National Research Service Award EY017507 (D.D.D.), and the National Institute of Mental Health Intramural Research Program (C.I.B.) We thank Jonas Kubilius for help with data collection and analyses, Brian Wandell for suggesting experiment 2, and Aniruddha Das, David Ferster, Ken Nakayama, Mriganka Sur, Frank Tong, and Gina Turrigiano for helpful comments on this manuscript.

Correspondence should be addressed to Daniel D. Dilks, McGovern Institute for Brain Research, Massachusetts Institute of Technology, 46-4141, Cambridge, MA 02139. E-mail: dilks@mit.edu.

D01:10.1523/JNEUROSCI.1557-09.2009

Copyright $\odot 2009$ Society for Neuroscience $\quad$ 0270-6474/09/298960-05\$15.00/0 sented in the lower LVF was perceived as a rectangle extending toward the upper LVF). Using functional magnetic resonance imaging (fMRI), Dilks et al. (2007) also observed concomitant changes in $\mathrm{V} 1$ and argued that the perceptual elongation is a consequence of the deprived "upper LVF" neurons now responding to stimuli that typically activate the adjacent "lower LVF." However, this study was conducted several months after the patient's stroke, providing no information about the time course of such changes. The question addressed here is whether perceptual elongation can be observed in healthy participants after reversible cortical deprivation and how quickly it occurs. Determining the timing of perceptual elongation will provide important constraints on the potential underlying mechanism.

We reversibly deprived input to a region of $\mathrm{V} 1$ in healthy human adults by patching one eye. The logic is as follows. In the healthy visual system, the cortical representation of the blind spot (BS) of the right eye receives information from the left eye only (and vice versa). Therefore, if the left eye is patched, the cortex corresponding to the BS of the right eye is deprived of its normal bottom-up input. To test for perceptual distortions, at different time points after deprivation, rectangular stimuli were presented one at a time around the "deprived" BS (see Fig. 1A) and participants judged the width or height of the stimuli. We found that, after only seconds of deprivation, rectangles adjacent to the deprived BS were perceived to be elongated toward the BS. We hypothesize these perceptual elongations are a consequence of rapid receptive field expansion of V1 neurons after deprivation, as reported in electrophysiological studies (Chino et al., 1992; Fiorani Júnior et al., 1992; Schmid et al., 1996; Calford et al., 2000) and call them "referred visual sensations" (RVS). 


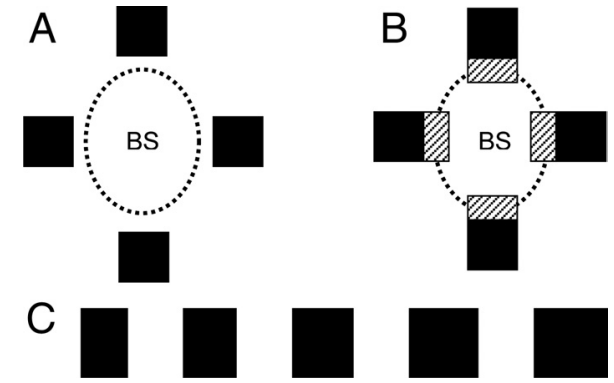

Figure 1. Design, predictions, and stimuli. $\boldsymbol{A}$, Tested positions (left, right, top, and bottom) for the width and height judgment tasks. $\boldsymbol{B}$, Predicted elongation for the deprived condition. If deprived cortex representing the $B S$ starts to respond to stimuli outside the $B S$, stimuli might be perceived as extending into the BS. C, Stimuli used for the width judgment task. Five rectangles with identical height $\left(2^{\circ}\right)$, but varying in width $\left(1.5,1.75,2,2.25\right.$, and $\left.2.5^{\circ}\right)$, were presented one at a time in each of the four locations. In the height judgment task, the rectangles were rotated $90^{\circ}$ (not pictured).

\section{Materials and Methods}

Participants. Forty-eight healthy individuals aged $19-50$ years (10 in experiment 1,5 in experiment 2, 9 in experiment 3, 20 in experiment 4 , and 4 in experiment 5). All participants gave informed consent. All had normal or corrected-to-normal visual acuity and were naive to the purpose of the experiment.

Blind spot localization. Each participants' left eye was patched using a $2 \times 3$ inch adhesive eye occlusor with a black eye patch placed over the top and were situated comfortably in a chinrest. We instructed participants to keep their right, unpatched eye fixated on a cross $\left(1^{\circ} \times 1^{\circ}\right)$ on the left edge of the computer screen. After fixating, a square $\left(0.5^{\circ} \times 0.5^{\circ}\right)$ first appeared $12^{\circ}$ right of the fixation cross (outside the left border of the average-sized BS). The square stimulus was white (luminance of $80 \mathrm{~cd} /$ $\mathrm{m}^{2}$ ) presented on a black (luminance of $1.1 \mathrm{~cd} / \mathrm{m}^{2}$ ) background. To obtain a rough right boundary, participants moved the square starting at the left of the BS (using the arrow keys) in $1^{\circ}$ increments to the right (through their BS) until the object first reappeared. At this point, the participant was instructed to press the space bar, and this boundary was recorded. Participants repeated this procedure for the left, top, and bottom boundaries to establish rough estimates for each position. These "rough" boundaries were then used to calculate the midpoint of the BS that was used for more precise measurements. To obtain more precise boundaries, participants followed the same procedure for multiple trials but with the white square beginning at the midpoint of the BS (not seen by the participant) and moving outward. Participants moved the square in increments of $0.25^{\circ}$ until the square reappeared at the edge of their BS in the respective direction. The order of the four directions was randomized. A total of three measurements was taken per direction and averaged to produce the four boundaries of the BS. Estimates of the borders of the BS using this method were highly reproducible.

Experiments 1-5. In the experimental tasks, participants were asked to judge the width or height of rectangles presented one at a time in each of four locations (left, right, top, and bottom) around the BS with $0.5^{\circ}$ spacing between the closest edge of the rectangle and the calculated border of the BS (to ensure visibility of this edge) (Fig. $1 \mathrm{~A}$ ). The rectangle stimuli were white (luminance of $80 \mathrm{~cd} / \mathrm{m}^{2}$ ) presented on a black (luminance of $1.1 \mathrm{~cd} / \mathrm{m}^{2}$ ) background. In the width judgment task, five rectangles with identical height $\left(2^{\circ}\right)$, but varying in width $(1.5,1.75,2,2.25$, and $2.5^{\circ}$ ), were presented in each of the four locations (Fig. $1 C$ ). In the height judgment task, the height and width dimensions were reversed. Each rectangle was presented eight times in each location for $150 \mathrm{~ms}$ (to avoid potential eye movements). In experiment 2 , only the right position was tested, and stimuli were presented at four distances $(0.5,1.5,2.5$, and $3.5^{\circ}$ ) from this position. Participants performed the horizontal judgment task. While keeping their unpatched eye on the fixation cross, participants were instructed to make the appropriate key response for "thinner/ shorter than a square," "square," or "wider/taller than a square" for the width and height judgment tasks, respectively. Unless otherwise noted, a binocular and monocular (right eye patched) control experiment was completed for each participant following the same procedures usually 1 week after the experimental testing. In both control experiments, the right eye BS representation, around which stimuli are presented, is "undeprived."

Responses were coded by assigning a +1 to each wider/taller than a square response, $\mathrm{a}-1$ to each shorter/thinner than a square response, and a 0 to each square response. A mean judgment score for each stimulus size difference (in degrees of visual angle) was computed by averaging the coded responses across trials. A negative mean judgment score indicates a predominance of thinner/shorter than a square responses, whereas a positive score indicates a predominance of wider/taller than a square responses.

The method of probits was used to identify the point of subjective equality (PSE), that is, the $50 \%$ point on the psychometric function. A negative PSE indicates a distortion in the predicted direction, and a positive PSE indicates a distortion in the anti-predicted direction. Additionally, this analysis identified the $95 \%$ confidence interval around the PSE, and the amount of distortion was considered significantly different from 0 if the confidence interval did not include 0 . Because the method of probits requires a dichotomous-dependent variable (e.g., shorter vs taller response), the square responses were divided equally between the shorter/thinner and taller/wider response categories. This division mimics a two-alternative forced-choice procedure.

\section{Results}

In experiment 1 , participants were tested immediately after the BS localization procedure (10 min after patching) and again after $2 \mathrm{~h}$. Two undeprived control experiments were run on the same subjects 1 week after the deprived condition; in one, the right eye was patched, and in the other, neither eye was patched.

Our key finding is that, at both $10 \mathrm{~min}$ and $2 \mathrm{~h}$ after eye patching, participants perceived rectangles placed adjacent to the deprived BS to be elongated toward the BS. Thus, rectangles presented to the left and right of the BS appeared wider than they actually were, and rectangles presented above and below the BS appeared taller than they were (Fig. $1 B$ shows predictions; Fig. 2 shows results). In contrast, participants were accurate in judging the width and height of rectangles presented next to the BS in both undeprived conditions (Fig. 2). The magnitude of the perceptual elongation was quantified using a probit analysis to determine the PSE, that is, the aspect ratio of the stimulus rectangle that would be perceived as a square. This analysis revealed that, on average, rectangles $\sim 10 \%$ shorter or narrower than a square (PSE of -9\%) would be perceived as squares (Fig. 2, red rectangles) after both $10 \mathrm{~min}$ and $2 \mathrm{~h}$ of deprivation. Interestingly, additional analyses revealed that the magnitude of elongation increased with increasing eccentricity: elongation was greatest on the right side of the BS, the most eccentric position tested, and weakest on the left side of the BS, closest to the fovea (supplemental Fig. 1, available at www.jneurosci.org as supplemental material). In three additional participants with deprivation extending to $9 \mathrm{~h}$, the magnitude of elongation was the same as that observed after 10 min (supplemental Fig. 2, available at www.jneurosci.org as supplemental material).

Next, we examined the magnitude of elongation as a function of distance of the stimulus from the border of the BS. In experiment 2, we presented stimuli at distances of $0.5,1.5,2.5$, and 3.5 from the most peripheral (right side) border of the BS after 10 min of deprivation. The magnitude of elongation decreased with increasing distance from the border of the deprived BS, with the greatest amount of distortion for stimuli closest to the BS (supplemental Fig. 3, available at www.jneurosci.org as supplemental material). These findings rule out the possibility that our elongations are attributable to the reduced acuity for peripheral stimuli: 


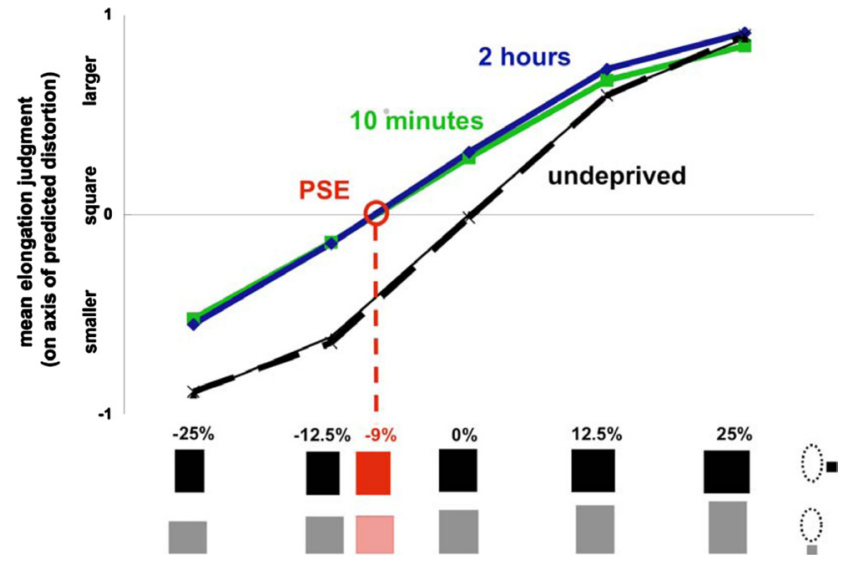

Figure 2. Perceptual elongations after $10 \mathrm{~min}$ and $2 \mathrm{~h}$ of deprivation. The figure depicts the mean elongation judgment along the axis of predicted distortion (i.e., the horizontal axis for stimuli presented to the left and right of the BS; the vertical axis for stimuli presented above and below the BS), as a function of stimulus aspect ratio (see black and gray rectangles). The black lines show the undeprived conditions (solid black line, right eye was patched; dashed black line, neither eye patched, i.e., binocular viewing). The blue and green lines show the deprived conditions at $2 \mathrm{~h}$ and $10 \mathrm{~min}$, respectively. In the undeprived control conditions, a square ( $x$-axis: $0 \%$ difference in width and height) is perceived veridically ( $y$-axis: 0 elongation judgment or square). In the deprived condition, this same stimulus is seen as elongated along the predicted axis of distortion (i.e., wider when presented to the left and right of the $B S$, and taller when presented to above and below the BS). More generally, the upward shift of the curves in the deprived conditions (blue and green lines) indicates an elongation of all rectangles along the axis of predicted distortion after $2 \mathrm{~h}$ of deprivation and after $10 \mathrm{~min}$. The PSE indicates the rectangles that were perceived as squares after deprivation (shown in red): the PSE was $-9 \%$, which indicates that a rectangle either $9 \%$ thinner than a square (if presented to the left and right of the BS) or $9 \%$ shorter than a square (if presented above and below the BS) was perceived as a square. The $9 \%$ magnitude of elongation was significantly different from 0 (in which 0 indicates no distortion and was outside the $95 \%$ confidence interval around the PSE) after both $10 \mathrm{~min}$ and $2 \mathrm{~h}$ of deprivation, and the magnitude of the effect did not differ in the two cases $\left(t_{(9)}=0.35 ; p=0.73\right)$. Moreover, the effect was significantly greater after each deprived condition than in each undeprived control condition $\left(t_{(9)}=4.54,3.36\right.$ for $10 \mathrm{~min}$ vs binocular and monocular controls, respectively, and $6.24,4.81$ for $2 \mathrm{~h}$ vs binocular and monocular controls, respectively; all $4 p$ values $<0.01$ ). No significant elongation was found in either of the undeprived control conditions (the 95\% confidence interval around the PSE for each condition included 0 ), nor did the two conditions differ from each other $\left(t_{(9)}=0.51 ; p=0.62\right)$.

although the $3.5^{\circ}$ stimuli were the farthest from fixation, shape judgments were more veridical ( $0 \%$ elongation) than those closer to the fovea.

Might the perceptual elongation reported here originate in the retina, for example, because of an anomaly in the tissue immediately adjacent to the optic disk? Neither of our undeprived control conditions provides a strong test of this hypothesis, because the test stimulus was never exclusively presented to the retina around the optic disk. In experiment 3, we therefore tested this hypothesis on five additional participants by presenting the test rectangles in one of four locations around the BS of the right eye (i.e., to the retinal position around the optic disk) but neither patching the left eye nor allowing it to see the test stimulus. Specifically, we placed a divider projecting from the nose toward the screen, thus obscuring the view of the stimulus by the left eye (without depriving it of input) and presenting the stimulus to the right eye only. If the critical factor in our elongation effect is deprivation of the cortical representation of the BS per se, rather than simply placement of the test stimulus next to the BS, we should observe no elongation in this case. Indeed, no elongation was found. Thus, elongation critically depends on cortical deprivation, i.e., the loss of bottom-up input (supplemental Fig. 4, available at www.jneurosci.org as supplemental material); simply

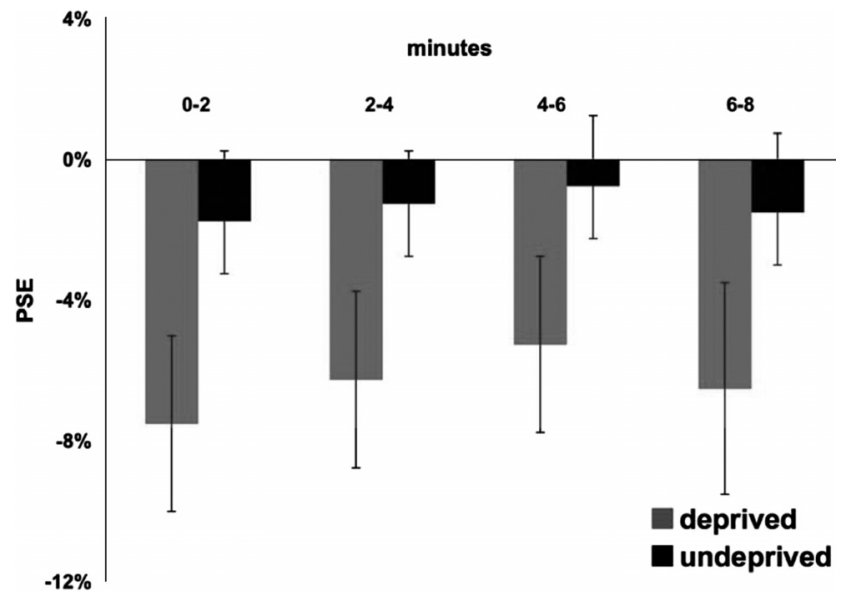

Figure 3. Time course of elongations over 8 min of deprivation. The graph depicts the PSEs (indicating the size rectangle perceived as square) for the deprived (gray bars) and undeprived (black bars) conditions across 2 min time intervals. Error bars reflect the $95 \%$ confidence intervals around the PSE. Within 2 min, rectangles presented next to the deprived BS had to be elongated by nearly $8 \%$ to be perceived as squares. In the deprived condition, the magnitude of elongation at all time intervals was significantly $>0$ (the $95 \%$ confidence interval around the PSEs did not include 0$)$, and they did not differ from each other $\left(F_{(3,57)}=0.164 ; p>0.9\right)$. In contrast, no significant elongation was found at any of the four time points in the undeprived condition (the $95 \%$ confidence intervals included 0 ), nor did they differ from each other $\left(F_{(3,57)}=1.35 ; p>0.25\right)$.

presenting stimuli to the retinal region adjacent to the BS without depriving the other eye is insufficient.

How quickly after deprivation does this elongation occur? In experiment 4, we asked whether perceptual elongation can occur in $<10 \mathrm{~min}$ of deprivation. Shape judgments as before were obtained over an 8 min period and analyzed in 2 min time bins. At all times points after patching, participants perceived rectangles adjacent to the deprived BS as elongated toward the BS (Fig. 3). Most strikingly, significant elongation was observed within 2 min: rectangles presented next to the BS had to be elongated by almost $10 \%$ to be perceived as squares (Fig. 3). In the undeprived condition (binocular), participants were accurate in judging the aspect ratio of rectangles at all time intervals.

Could this elongation occur within seconds? And what is the time course of "recovery"? To examine more finely the time courses of elongation and recovery, in experiment 5 , we obtained aspect ratio judgments over alternating 2 min blocks of deprivation and nondeprivation conditions and analyzed the data in $1 \mathrm{~s}$ bins for each condition. Specifically, individuals were asked to close both eyes, and, while they were closed, one eye was patched (taking $\sim 5 \mathrm{~s}$ ). With both eyes closed, the participant waited for the experimenter's instruction to open the unpatched eye, fixate, and immediately begin judging the width or height of the rectangles (deprived condition). After $2 \mathrm{~min}$, the fixation cross was removed, and the participant closed the unpatched eye. The experimenter then removed the patch while the participant continued to keep both eyes closed. After the removal of the patch, at the experimenter's instruction, the participant opened both eyes and immediately began the task (undeprived condition). This procedure was repeated 20 times ( 10 blocks of each condition). Even on the very first trial, which occurred within seconds of the onset of deprivation, participants perceived rectangles adjacent to the deprived BS as significantly elongated toward the BS (Fig. 4). Moreover, the elongations reversed within seconds when the deprivation ended, showing that recovery is equally rapid. 


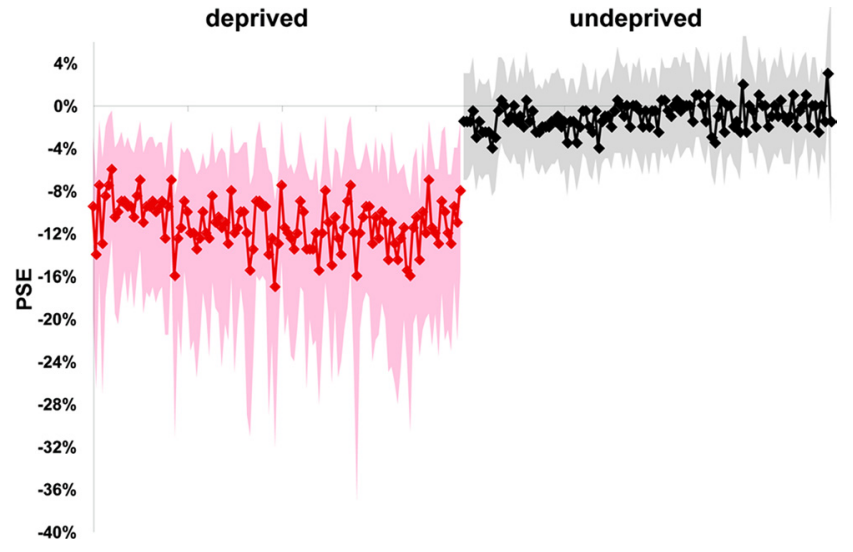

Figure 4. Second-by-second analysis of perceptual elongations. The figure depicts the PSES (indicating the size rectangle perceived as square) for every trial over a 2 min block for the deprived (red line) and undeprived (black line) conditions. Each trial took 1s. The shaded area around each curve indicates the $95 \%$ confidence interval around that PSE. On the very first trial (i.e., within the first second after the onset of deprivation), significant elongation was observed (PSE of $-10 \%$, and the $95 \%$ confidence interval did not include 0 ). In contrast, on the very first trial after the deprivation ends, the elongations reverse and perception returns to normal (PSE of $-2 \%$, and the $95 \%$ confidence interval includes 0 ).

\section{Discussion}

Using a noninvasive method to reversibly deprive a region of $\mathrm{V} 1$ (i.e., the cortical representation of the BS) in healthy human adults, we have shown perceptual elongations around the deprived BS, and, crucially, we found that these elongations occur within seconds of deprivation. Our findings suggest the following hypothesis: perceptual elongations are a consequence of rapid receptive field expansion after deprivation. For example, if the deprived V1 region representing the BS begins to respond to stimuli that normally activate adjacent cortex only, then stimuli presented adjacent to the deprived BS region will appear elongated toward that region. This hypothesis is consistent with electrophysiological studies demonstrating that, after minutes or hours of deprivation, deprived V1 neurons come to respond to stimuli that normally activate adjacent cortex only (Chino et al., 1992; Fiorani Júnior et al., 1992; Schmid et al., 1996; Calford et al., 2000). Moreover, the reported RVS is too fast to be the result of structural changes in the cortex (e.g., the growth of new connections) (Darian-Smith and Gilbert, 1994; Keck et al., 2008), instead implicating unmasking of preexisting connections as the underlying neural mechanism (Das and Gilbert, 1995; Calford et al., 2003). Note, however, that our effect occurs within seconds, faster than reported previously. Physiological studies have reported effects of deprivation occurring within minutes or hours of retinal lesioning but could not test for effects occurring within seconds because of widespread temporary deactivation of neurons immediately after the lesioning procedure (Chu et al., 1998). The extreme rapidity of our effect may also provide an explanation for one recent failure to find any evidence for reorganization (Smirnakis et al., 2005): the baseline measurement in that study was conducted hours after the retinal lesions were made, when the changes we observed would have already occurred.

It is an open question whether the proposed mechanism also underlies the other reported cases of long-term reorganization of visual cortex, i.e., the changes in retinotopic maps from patients with scotomas (Baker et al., 2005, 2007; Dilks et al., 2007, 2009; Masuda et al., 2008), and the changes in receptive fields that have been observed physiologically after months of deprivation (Kaas et al., 1990; Heinen and Skavenski, 1991; Gilbert and Wiesel,
1992). Perhaps structural changes in cortex also occur, as reported in mice (Keck et al., 2008) and in some (Darian-Smith and Gilbert, 1994) but not all (Horton and Hocking, 1998; Smirnakis et al., 2005) studies in other species and play a significant role in cortical reorganization over longer timescales (Kaas et al., 1990; Heinen and Skavenski, 1991; Gilbert and Wiesel, 1992; Baker et al., 2005, 2008; Dilks et al., 2007, 2009). As noted previously, a previous study showed perceptual elongations much like those shown here, along with corresponding changes in retinotopic organization in the same subject, suggesting that physiological changes in V1 receptive fields might also be involved in the distortion effects observed here (Dilks et al., 2007). The larger magnitude of elongation reported in the stroke patient with deprived V1 compared with the BS effects reported here may reflect the much larger scotoma in the former case.

Although our hypothesis implicates receptive field changes in the human adult, one might imagine that the elongations we observed could reflect reorganization occurring in infancy or childhood. For example, the BS could be described as a "lesion" from birth, whereby neurons in the region of the lateral geniculate nucleus (LGN) corresponding to the BS are deprived of any retinal input. This deprivation could result in reorganization in the LGN at an early developmental age, such that patching one eye simply uncovers this early subcortical change. However, previous research reported that no such reorganization occurs in the LGN except in the case of albinism (Guillery and Kaas, 1971, 1973; Guillery et al., 1984). Furthermore, in the normal visual system, retinotopy in the LGN is so precise that the BS is represented by small gaps free of cells (Kaas et al., 1972, 1973); thus, there are no neurons in LGN representing the BS to become responsive to inputs from adjacent retinal locations. These findings argue against the hypothesis that our results reflect reorganization in the LGN early in life.

Our findings are consistent with another study that tested for perceptual distortion resulting from an "artificial scotoma” (Kapadia et al., 1994) (see also Tailby and Metha, 2004), in which a region of retina sees only a uniform gray square, while the surrounding region is stimulated by dynamic white noise. This study reported that the perceived position of a short line segment presented near the border of the artificial scotoma was slightly shifted (2.50-5.00 min of arc) toward the scotoma. Furthermore, Kapadia et al. (1994) reported that the shift occurred within $2 \mathrm{~s}$ of exposure to the artificial scotoma display. This shift was attributed to receptive field expansion, as seen in the animal studies that found expansion of receptive fields in visual field locations corresponding to the artificial scotoma (Fiorani Júnior et al., 1992; Pettet and Gilbert, 1992; DeAngelis et al., 1995). Whether the elongations reported here and the mislocalizations from artificial scotomata involve similar mechanisms remains an open question. Note, however, that our effects are much larger (three to six times larger) than those reported previously by Kapadia et al. (1994). Moreover, it is unclear how artificial scotomas relate to visual deprivation because all areas of the retina are still receiving visual input, albeit uniform illumination. In our paradigm, patching effectively removed bottom-up input to a region of cortex, more closely corresponding to deprivation of input in the retinal lesion studies. In another study (Van Baelen et al., 2005), spatial mislocalizations were also found around the BS, but the time course was not investigated.

The perceptual elongation reported here might also bear some resemblance to filling-in of the BS, in which the entire BS appears to fill in with the texture or color surrounding it under monocular viewing conditions, and collinear bars that abut the BS are 
"completed" and perceived as continuous despite the gap. However, previous studies (Fiorani Júnior et al., 1992; Ramachandran, 1992; Awater et al., 2005; Matsumoto and Komatsu, 2005) have reported that no perceptual filling-in occurs when single bars are presented to just one side of the BS as used in this study. Thus, our effect cannot be explained by the traditional perspective on filling-in around the BS, although one appealingly parsimonious account would be that common mechanisms underlie both our distortion phenomena and filling-in around the BS. Unfortunately, even at the highest spatial resolution we could obtain (1 $\mathrm{mm}$ isotropic), we were unable to resolve fMRI responses to distinct subregions within the BS and so cannot (yet) test this hypothesis with fMRI (D. D. Dilks, unpublished data). Interestingly, however, two single-unit studies have reported weak neural filling-in when a single bar is presented to only one side of the BS (Fiorani Júnior et al., 1992; Matsumoto and Komatsu, 2005), providing a likely neural correlate of our perceptual effect.

In summary, we found perceptual elongation that occurs within seconds of visual deprivation, that lasts as long as the deprivation continues, and that reverses within seconds if the deprivation ends. We attribute this elongation to rapid changes in receptive field properties in early visual cortex, and the rapidity of this effect implicates unmasking of preexisting connections as the underlying neural mechanism. Our findings point to an important new set of questions for future investigation. Are the mechanisms underlying the hypothesized very rapid changes of visual processing observed here the same as those resulting from macular degeneration (Baker et al., 2005, 2008; Masuda et al., 2008; Dilks et al., 2009), stroke (Dilks et al., 2007), and retinal lesions (Kaas et al., 1990; Heinen and Skavenski, 1991; Gilbert and Wiesel, 1992; Darian-Smith and Gilbert, 1994)? Or, are additional mechanisms, perhaps including structural change in neural connections, involved in these longer-term phenomena? Are these phenomena related to, or entirely distinct from, the neural mechanisms underlying developmental plasticity? Whatever the ultimate answers to these questions, our results highlight the stunning ability of the cortex to adapt moment to moment to changes in experience even in adulthood.

\section{References}

Awater H, Kerlin JR, Evans KK, Tong F (2005) Cortical representation of space around the blind spot. J Neurophysiol 94:3314-3324.

Baker CI, Peli E, Knouf N, Kanwisher NG (2005) Reorganization of visual processing in macular degeneration. J Neurosci 25:614-618.

Baker CI, Dilks DD, Peli E, Kanwisher N (2008) Reorganization of visual processing in macular degeneration: replication and clues about the role of foveal loss. Vision Res 48:1910-1919.

Borsook D, Becerra L, Fishman S, Edwards A, Jennings CL, Stojanovic M, Papinicolas L, Ramachandran VS, Gonzalez RG, Breiter H (1998) Acute plasticity in the human somatosensory cortex following amputation. Neuroreport 9:1013-1017.

Calford MB (2002) Dynamic representational plasticity in sensory cortex. Neuroscience 111:709-738.

Calford MB, Wang C, Taglianetti V, Waleszczyk WJ, Burke W, Dreher B (2000) Plasticity in adult cat visual cortex (area 17) following circumscribed monocular lesions of all retinal layers. J Physiol 524:587-602.

Calford MB, Wright LL, Metha AB, Taglianetti V (2003) Topographic plasticity in primary visual cortex is mediated by local corticocortical connections. J Neurosci 23:6434-6442.

Chino YM, Kaas JH, Smith EL 3rd, Langston AL, Cheng H (1992) Rapid reorganization of cortical maps in adult cats following restricted deafferentation in retina. Vision Res 32:789-796.

Chu Y, Humphrey MF, Alder VV, Constable IJ (1998) Immunocytochemical localization of basic fibroblast growth factor and glial fibrillary acidic protein after laser photocoagulation in the Royal College of Surgeons rat. Aust N Z J Ophthalmol 26:87-96.
Darian-Smith C, Gilbert CD (1994) Axonal sprouting accompanies functional reorganization in adult cat striate cortex. Nature 368:737-740.

Das A, Gilbert CD (1995) Receptive field expansion in adult visual cortex is linked to dynamic changes in strength of cortical connections. J Neurophysiol 74:779-792.

DeAngelis GC, Anzai A, Ohzawa I, Freeman RD (1995) Receptive field structure in the visual cortex: does selective stimulation induce plasticity? Proc Natl Acad Sci U S A 92:9682-9686.

Dilks DD, Serences JT, Rosenau BJ, Yantis S, McCloskey M (2007) Human adult cortical reorganization and consequent visual distortion. J Neurosci 27:9585-9594.

Dilks DD, Baker CI, Peli E, Kanwisher N (2009) Reorganization of visual processing in macular degeneration is not specific to the "preferred retinal locus." J Neurosci 29:2768-2773.

Fiorani Júnior M, Rosa MG, Gattass R, Rocha-Miranda CE (1992) Dynamic surrounds of receptive fields in primate striate cortex: a physiological basis for perceptual completion? Proc Natl Acad Sci U S A 89:8547-8551.

Gilbert CD, Wiesel TN (1992) Receptive field dynamics in adult primary visual cortex. Nature 356:150-152

Guillery RW, Kaas JH (1971) A study of normal and congenitally abnormal retinogeniculate projections in cats. J Comp Neurol 143:73-100.

Guillery RW, Kaas JH (1973) Genetic abnormality of the visual pathways in a "white" tiger. Science 180:1287-1289.

Guillery RW, Hickey TL, Kaas JH, Felleman DJ, Debruyn EJ, Sparks DL (1984) Abnormal central visual pathways in the brain of an albino green monkey (Cercopithecus aethiops). J Comp Neurol 226:165-183.

Heinen SJ, Skavenski AA (1991) Recovery of visual responses in foveal V1 neurons following bilateral foveal lesions in adult monkey. Exp Brain Res 83:670-674.

Horton JC, Hocking DR (1998) Monocular core zones and binocular border strips in primate striate cortex revealed by the contrasting effects of enucleation, eyelid suture, and retinal laser lesions on cytochrome oxidase activity. J Neurosci 18:5433-5455.

Kaas JH, Guillery RW, Allman JM (1972) Some principles of organization in the dorsal lateral geniculate nucleus. Brain Behav Evol 6:253-299.

Kaas JH, Guillery RW, Allman JM (1973) Discontinuities in the dorsal lateral geniculate nucleus corresponding to the optic disc: a comparative study. J Comp Neurol 147:163-179.

Kaas JH, Krubitzer LA, Chino YM, Langston AL, Polley EH, Blair N (1990) Reorganization of retinotopic cortical maps in adult mammals after lesions of the retina. Science 248:229-231.

Kapadia MK, Gilbert CD, Westheimer G (1994) A quantative measure for short-term cortical plasticity in human vision. J Neurosci 14:451-457.

Keck T, Mrsic-Flogel TD, Vaz Afonso M, Eysel UT, Bonhoeffer T, Hübener M (2008) Massive restructuring of neuronal circuits during functional reorganization of adult visual cortex. Nat Neurosci 11:1162-1167.

Masuda Y, Dumoulin SO, Nakadomari S, Wandell BA (2008) V1 projection zone signals in human macular degeneration depend on task, not stimulus. Cereb Cortex 18:2483-2493.

Matsumoto M, Komatsu H (2005) Neural responses in the macaque v1 to bar stimuli with various lengths presented on the blind spot. J Neurophysiol 93:2374-2387.

Pettet MW, Gilbert CD (1992) Dynamic changes in receptive-field size in cat primary visual cortex. Proc Natl Acad Sci U S A 89:8366-8370.

Ramachandran VS (1992) Filling in the blind spot. Nature 356:115.

Ramachandran VS, Rogers-Ramachandran D, Stewart M (1992) Perceptual correlates of massive cortical reorganization. Science 258:1159-1160.

Schmid LM, Rosa MG, Calford MB, Ambler JS (1996) Visuotopic reorganization in the primary visual cortex of adult cats following monocular and binocular retinal lesions. Cereb Cortex 6:388-405.

Smirnakis SM, Brewer AA, Schmid MC, Tolias AS, Schüz A, Augath M, Inhoffen W, Wandell BA, Logothetis NK (2005) Lack of long-term cortical reorganization after macaque retinal lesions. Nature 435:300-307.

Tailby C, Metha A (2004) Artificial scotoma-induced perceptual distortions are orientation dependent and short lived. Vis Neurosci 21:79-87.

Van Baelen M, Claessens P, Stalmans P, Wagemans J (2005) Perceptual distortions in the visual field surrounding a scotoma: psychophysical measurement with a "spatial interval discrimination task." Int Congr Ser 1282:749-753.

Weiss T, Miltner WH, Liepert J, Meissner W, Taub E (2004) Rapid functional plasticity in the primary somatomotor cortex and perceptual changes after nerve block. Eur J Neurosci 20:3413-3423. 